\title{
Originales
}

\section{Eficacia y eficiencia de una Unidad de Medicina Intensiva polivalente}

\author{
F. GARCÍA LIZANA, J.L. MANZANO ALONSO*, E. TREVIÑO PEÑA, C. PÉREZ ORTIZ, I. GARCÍA OLIVA, \\ A. VALLEJO BÁEZ Y P. SAAVEDRA SANTANA
}

Unidades de Medicina Intensiva. Hospital Ntra. Sra. del Pino* y Hospital Universitario Insular de Las Palmas de Gran Canaria. Departamento de Matemáticas. Universidad de Las Palmas de Gran Canaria.

Fundamento. Al médico se le exige continuamente mejorar los cuidados de salud y contener el gasto. Por ello necesitamos conocer nuestros resultados (de mortalidad y calidad de vida [CDV]) así como los recursos invertidos para poder mejorar la eficacia y la eficiencia de nuestra Unidad de Medicina Intensiva (UMI) en el futuro.

Método. Estudio retrospectivo de 1.000 pacientes ingresados sucesivamente en una UMI polivalente. Se excluyen menores de 15 años, extranjeros y reingresos. Se recogieron datos demográficos, antecedentes personales, diagnóstico, fracaso orgánico, supervivencia en UMI, hospitalaria y al año, CDV previa y al año (con el EuroQOL modificado) y los costes según los días de estancia en el hospital y en la UMI. Se utilizó el test de Kruskal-Wallis para variables numéricas.

Resultados. De los 843 pacientes incluidos en el estudio, 212 (25\%) fallecieron en la unidad, 64 $(7,5 \%)$ en el hospital y $64(7,5 \%)$ durante el año de seguimiento. De los 503 supervivientes al año, el 58\% gozaban de buen estado de salud, el $30 \%$ presentaban alguna discapacidad pero vivían independientemente y sólo un $11 \%$ tenían discapacidades severas. Los pacientes que costaron significativamente menos fueron los que se recuperaron totalmente. El $23 \%$ de los pacientes consumió el $70 \%$ de todos los recursos, y éstos fueron los pacientes que fallecieron y los que quedaron con peor CDV.

Conclusión. El $60 \%$ de los pacientes sobrevivían al año, y de ellos el $89 \%$ tenían una CDV

\footnotetext{
Correspondencia: Dra. F. García Lizana.

Unidad de Medicina Intensiva.

Hospital Universitario Insular de Gran Canaria.

C/ Dr. Pasteur s/n.

35016 Las Palmas de Gran Canaria.

Manuscrito aceptado el 14-XII-1999.
}

aceptable, lo cual demuestra la eficacia de las UMI y justifica su existencia. Los pacientes que fallecieron o quedaron con una CDV más limitada consumieron la mayor parte de los recursos. Sólo cuando nuestra capacidad predictiva de mortalidad y CDV sea correcta en el $100 \%$ de los casos y podamos limitar el tratamiento, mejoraremos la eficiencia. Mientras tanto sólo la sociedad puede marcar la directriz en la distribución y contención del gasto.

PALABRAS CLAVE: calidad de vida, costes, limitación y retirada de soporte vital, Medicina Intensiva.

\section{EFFECTIVENESS AND EFFICIENCY IN A MULTI- DISCIPLINARY ICU}

Background. Physicians are constantly requested to improve health' care and the costs of their therapies. That is the reason why we need to know our results (in terms of death and quality of life (QOL) and the resources consumed for improving the effectiveness and efficiency of our Intensive Care Units in a future.

Method. Retrospective descriptive study of 1000 patients, admitted to a multidisciplinary ICU. We excluded people younger than 15 years, foreigners and readmitted patients. Data regarding demography, previous diseases, diagnoses at admission, organic failure, ICU survival, hospital and one year later survival, previous and one year later QOL (with the modified EuroQOL) and the costs related to the length of stay in the hospital and ICU were collected. Kruskall-Wallis test was used for numerical variables.

Results. 843 patients were included in the study. $212(25 \%)$ died in the unit, $64(7.5 \%)$ died in the hospital and $64(7.5 \%)$ during the following 
year. From the total of 503 one year surviving patients, $58 \%$ showed a good health status, $30 \%$ suffered some discapacity but they live in a independent way and only the $11 \%$ had severe discapacities. The patients whose costs were lower were those that completely improved $23 \%$ of the patients consumed the $70 \%$ of global resources, and those were the patients that died or remained with the worst QOL.

Conclusion. $60 \%$ of the patients survived one year later, and $89 \%$ of them with a good QOL, a fact which shows the ICU effectiveness and justified their existence. The patients who died or remained with a more limited $\mathrm{QOL}$ consumed the highest proportion of resources. Only if our predictive capacity of mortality and QOL becomes correct in $100 \%$ of cases we would be able to limit the treatments, and we shall improve the effectiveness. Meanwhile, only the Society can detemine the distribution and contention of costs.

KEY WORDS: Quality of life costs, withholding and withdrawing life-sustaining therapy, Intensive Care.

(Med Intensiva 2000; 24: 211-219)

\section{INTRODUCCIÓN}

Las Unidades de Medicina Intensiva (UMI) son áreas de los hospitales modernos dotadas de alta tecnología que consumen del $3 \%$ al $20 \%$ del coste hospitalario $^{1}$ y debido al aumento de la población anciana se estima que este gasto ascienda considerablemente. Es evidente que existen pacientes en los que los cuidados intensivos sólo prolongan el proceso de muerte, y puesto que los recursos son limitados es imprescindible conocer nuestros resultados y el coste invertido en ellos para poder hacer un balance correcto del coste-beneficio y poder mejorar la eficacia y la eficiencia de estas unidades. El beneficio de toda actividad médica, y concretamente en la UMI, se mide en términos de mortalidad, ganancias de calidad de vida (CDV) o ganancias sociales (lo que la sociedad recibe al incorporarse al trabajo los pacientes rehabilitados), de ahi que nuestro objetivo ha sido evaluar la mortalidad, la CDV y la situación laboral al año del alta de UMI en relación con su coste.

\section{MATERIAL Y MÉTODOS}

Estudiamos retrospectivamente a 1.000 pacientes ingresados sucesivamente en nuestra UMI durante un período de 18 meses. Fueron excluidos del estudio los pacientes menores de 15 años y los pacientes extranjeros que fueron trasladados a su país de origen. De los pacientes que reingresaron en la UMI sólo se consideró su primer ingreso.

Se recogieron los datos de filiación, edad, sexo, estancia hospitalaria y en UMI, antecedentes personales, diagnóstico principal que motivó el ingreso en la UMI, insuficiencia orgánica durante su estancia en la misma, mortalidad en UMI, hospitalaria y al año del alta de UMI. También se estudió la actividad laboral y CDV previa al ingreso (CDVp) y al año del alta de la unidad.

Los antecedentes personales considerados fueron: enfermedades cardiovasculares, endocrinometabólicas, gastrointestinales, respiratorias, neurológicas, renales, psiquiátricas, cirugía cardíaca y miscelánea. Para su análisis estadístico se utilizó en cada paciente la suma de todos ellos (SAP). Los grupos diagnósticos considerados se muestran en la tabla 1. La gravedad de la enfermedad que motivó el ingreso se evaluó por el número de órganos afectados (NOF) y los criterios de fracaso orgánico ${ }^{2-4}$ se muestran el la tabla 2. Los pacientes se clasificaron en cuatro grupos de gravedad: (1) sin fracaso orgánico; (2) fracaso de un órgano; (3) fracaso de dos y (4) fracaso de más de dos órganos.

Para conocer la CDV de los supervivientes al año de haber sido dados de alta de la UMI (CDVa) y tres meses antes de su ingreso (CDVp), se elaboró un cuestionario (Apéndice 1) basado en el cuestionario del EuroQOL ${ }^{5,6}$ al que añadimos aspectos calificadores del estado laboral del paciente antes y después de su ingreso en la UMI, a fin de interpretar la influencia de la enfermedad en este aspecto. Este cuestionario se completó mediante entrevista telefónica o por correo directamente con los pacientes o sus familiares más cercanos. Se establecieron tres categorías de CDV (Apéndice 1): A: gozan de buen estado de salud, B: presentan algunas discapacidades pero viven independientemente; $\mathrm{C}$ : dependen de los demás para sobrevivir.

Los costes que ocasionó cada paciente que había ingresado en la UMI se obtuvieron a partir del coste de una cama/día de estancia. El valor se extrajo de los datos de la Administración del Hospital Ntra. Sra. del Pino, obteniéndose un coste de cama/día de UMI de 180.000 pesetas (1.084 euros) y un coste medio de cama/día de sala de hospitalización de 35.000 pesetas (211 euros). En ellos se incluye los gastos de personal, material fungible, farmacia, amortización del equipo, etc. La estancia en UMI corresponde al número de días que permaneció ingresado un enfermo en la unidad. Las estancias de menos de 24 horas fueron consideradas de un día completo. La estancia hospitalaria corresponde al número de días que permaneció el paciente en el hospital, incluida la estancia de UMI. Sin embargo, para el cálculo del coste de la planta se excluyó la estancia de UMI.

Las variables numéricas se expresan en medias (DE) y en percentiles y las categóricas en tablas de frecuencias. Se utilizó el test de Kruskal-Wallis para las variables numéricas $(\mathrm{p}<0,05)$.

\section{RESULTADOS}

De los 1.000 pacientes estudiados se excluyeron 157: $75(7,3 \%)$ eran reingresos, 4 pacientes eran menores de 15 años, 16 eran extranjeros y 62 fueron ilocalizables. 
TABLA 1. Características demográficas y clínicas de la población estudiada

\begin{tabular}{|c|c|c|c|c|c|}
\hline & \multirow[b]{2}{*}{$\begin{array}{l}\text { Fallecidos UMI } \\
\quad \mathrm{N}(\%) \\
212(25,1)\end{array}$} & \multirow[b]{2}{*}{$\begin{array}{c}\text { Fallecidos post-UMI } \\
\mathrm{N}(\%) \\
128(15,2)\end{array}$} & \multicolumn{3}{|c|}{ Categorías de calidad de vida al año (CDVa) } \\
\hline & & & $\begin{array}{c}\mathrm{A} \\
\mathrm{N}(\%) \\
293(58,2)\end{array}$ & $\begin{array}{c}\mathrm{B} \\
\mathrm{N}(\%) \\
154(30,6)\end{array}$ & $\begin{array}{c}\mathrm{C} \\
\mathrm{N}(\%) \\
56(11,1)\end{array}$ \\
\hline \multicolumn{6}{|l|}{ CDV previa (CDVp): } \\
\hline A & & & $158(53,9)$ & $81(52,6)$ & $28(50)$ \\
\hline B & & & $129(44)$ & $64(41,6)$ & $15(26,8)$ \\
\hline $\mathrm{C}$ & & & $6(2)$ & $9(5,8)$ & $13(23,2)$ \\
\hline \multicolumn{6}{|l|}{ Sexo: } \\
\hline Hombre & $143(67,5)$ & $82(64)$ & $213(72,7)$ & $116(75,3)$ & $40(71,4)$ \\
\hline Mujer & $69(32,5)$ & $46(36)$ & $80(27,3)$ & $38(24,7)$ & $16(28,6)$ \\
\hline \multicolumn{6}{|l|}{ Número de órganos fracasados: } \\
\hline Ninguno & & $32(25)$ & $141(48,1)$ & $49(31,8)$ & $13(23,2)$ \\
\hline 1 & $5(2,4)$ & $31(24)$ & $63(21,5)$ & $42(27,3)$ & $15(26,8)$ \\
\hline 2 & $63(29,7)$ & $36(28)$ & $63(21,5)$ & $35(22,7)$ & $16(28,6)$ \\
\hline$<2$ & $144(67,9)$ & $29(23)$ & $26(8,9)$ & $28(18,2)$ & $12(21,4)$ \\
\hline \multicolumn{6}{|l|}{ Diagnóstico: } \\
\hline Cirugía cardíaca & $17(8)$ & $10(7,8)$ & $104(35,6)$ & $36(23,4)$ & $4(7,1)$ \\
\hline Enfermedad cardiovascular & $48(22,6)$ & $39(30,5)$ & $102(34,9)$ & $41(26,6)$ & $8(14,3)$ \\
\hline PCR & $12(5,7)$ & $8(6,3)$ & $4(1,4)$ & $2(1,3)$ & \\
\hline Cirugía vascular & $8(3,8)$ & $3(2,3)$ & $11(3,7)$ & $5(3,2)$ & $5(8,9)$ \\
\hline Cirugía de tórax & $6(2,8)$ & $7(5,5)$ & $15(5,1)$ & $6(3,9)$ & $2(3,6)$ \\
\hline Enfermedad respiratoria & $34(16)$ & $20(15,6)$ & $13(4,5)$ & $13(8,4)$ & $4(7,1)$ \\
\hline Enfermedad neurológica & & & & & \\
\hline (médica o quirúrgica) & $29(13,7)$ & $11(8,6)$ & $9(3,1)$ & $11(7,1)$ & $4(7,1)$ \\
\hline Enfermedad del aparato digestivo & $22(10,4)$ & $16(12,5)$ & $5(1,7)$ & $6(3,9)$ & $5(8,9)$ \\
\hline Traumatismos & $2(0,9)$ & $2(1,6)$ & $5(1,7)$ & $8(5,2)$ & $4(7,1)$ \\
\hline TCE & $13(6,1)$ & $2(1,6)$ & $10(3,4)$ & $15(9,7)$ & $12(21,4)$ \\
\hline Sepsis & $5(2,4)$ & $3(2,3)$ & $5(1,7)$ & $5(3,2)$ & \\
\hline Miscelánea & $16(7,5)$ & $7(5,5)$ & $10(3,4)$ & $6(3,9)$ & $2(3,6)$ \\
\hline \multicolumn{6}{|l|}{ SAP: } \\
\hline 0 & & & & & \\
\hline 1 & $34(16)$ & $10(7,8)$ & $48(16,4)$ & $30(19,5)$ & $14(25)$ \\
\hline 2 & $81(38,2)$ & $40(31,3)$ & $135(46,1)$ & $60(39)$ & $20(35,7)$ \\
\hline 3 & $65(30,6)$ & $56(43,8)$ & $90(30,7)$ & $47(30,5)$ & $16(28,6)$ \\
\hline 4 & $28(13,2)$ & $19(14,8)$ & $20(6,8)$ & $15(9,7)$ & $5(8,9)$ \\
\hline 5 & $4(1,9)$ & $2(1,6)$ & & $2(1,3)$ & $1(1,8)$ \\
\hline & & $1(0,8)$ & & & \\
\hline \multicolumn{6}{|l|}{ Grupos de edad (años) } \\
\hline $15-44$ & $37(17,5)$ & $10(7,8)$ & $66(22,5)$ & $45(29,2)$ & $11(19,6)$ \\
\hline $45-64$ & $80(37,8)$ & $52(40,6)$ & $138(47,1)$ & $72(46,8)$ & $19(33,9)$ \\
\hline$\geq 65$ & $95(44,8)$ & $66(51,6)$ & $89(30,4)$ & $37(24)$ & $26(46,4)$ \\
\hline Edad media (años) & $59,28(15,98)$ & $62,91(12,66)$ & $54,91(15,65)$ & $52,87(16,73)$ & $59,41(17,98)$ \\
\hline \multicolumn{6}{|l|}{ Estancia UMI (días) } \\
\hline Med (DE) & $12,70(24,82)$ & $12,22(22,99)$ & $5,45(6,19)$ & $9,95(12,13)$ & $18,62(23,58)$ \\
\hline Min y máx & $1-257$ & $1-185$ & $1-43$ & $1-66$ & $1-127$ \\
\hline \multicolumn{6}{|l|}{ Estancia hospital (días) } \\
\hline Med (DE) & $19,04(29,02)$ & $39,34(42,02)$ & $23,88(19,81)$ & $35,37(35,33)$ & $64,25(71,65)$ \\
\hline Min y máx & $1-263$ & $2-300$ & $3-140$ & $4-253$ & $4-449$ \\
\hline
\end{tabular}

A: buen estado de salud; B: alguna discapacidad pero independientes; C: dependen de los demás; TCE: traumatismo craneoencefálico; SAP: suma de antecedentes personales; UMI: Unidad de Medicina Intensiva; PCR: parada cardiorrespiratoria; CDV: calidad de vida.

De los 843 pacientes estudiados, el 70,5\% (594) eran varones y el $29,5 \%$ (249) mujeres y la edad media fue de 57,15 (16,02); (rango 15-93). La estancia media en UMI fue de $10(18,03)$ y la hospitalaria de $29,79(36,27)$. En el $28 \%$ de los pacientes no fracasó ningún órgano, en el $18,5 \%$ uno, en un $25 \%$ dos y en un $28 \%$ más de dos órganos. Las enfermedades correspondientes a los 843 pacientes estudiados se distribuyeron de la siguiente forma: un $20,3 \%$ (171) fueron postoperados cardíacos, 28,2\% (238) presentaron enfermedad cardiovascular, $10 \%$ tenían patología respiratoria, un $8,3 \%$ enfermedades neuroquirúrgicas, el $6,4 \%$ presentaba patología digestiva y el $6,2 \%$ traumatismo craneoencefálico (TCE). El resto se distribuyeron entre otras patologías. Las características clínicas y demográficas de los pacientes estudiados según su evolución y CDV se muestran en la tabla 1.

El 40,3\% de los 843 pacientes habían fallecido al año (212 pacientes lo hicieron en la Unidad de Cuidados Intensivos [UCI], 64 en planta de hospitalización y 64 durante el seguimiento). De los 503 supervivien- 
TABLA 2. Criterios de fracaso orgánico

\begin{tabular}{|c|c|}
\hline $\begin{array}{l}\text { Fracaso } \\
\text { cardiovascular } \\
\text { (Presencia de uno o } \\
\quad \text { más de lo siguiente) }\end{array}$ & $\begin{array}{l}\text { Fallo ventricular o arritmia } \\
\text { (taquicardia o fibrilación) no } \\
\text { existentes previamente, } \\
\text { necesidad de drogas } \\
\text { vasoactivas } \\
\text { o antiarrítmicos, frecuencia } \\
\text { cardíaca }<50 \text { latidos/minuto, } \\
\text { tensión arterial media }<50 \\
\text { mmHg }\end{array}$ \\
\hline $\begin{array}{l}\text { Fracaso respiratorio } \\
\text { (Presencia de uno o } \\
\text { más de lo siguiente) }\end{array}$ & $\begin{array}{l}\text { Necesidad de ventilación } \\
\text { mecánica, frecuencia respiratoria } \\
<5 / \text { min o > 49/min } \mathrm{PaCO}_{2}>50 \\
\mathrm{mmHg} \text {, D (A-a) } \mathrm{O}_{2}>350 \mathrm{mmHg}\end{array}$ \\
\hline $\begin{array}{l}\text { Fracaso renal } \\
\text { (Presencia de uno o } \\
\quad \text { más de lo siguiente) }\end{array}$ & $\begin{array}{l}\text { Creatinina }>2,3 \mathrm{~g} / \mathrm{dl} \text {, urea }>215 \\
\mathrm{mg} / \mathrm{dl} \text {, diuresis }<479 \mathrm{ml} / 24 \mathrm{~h} \text {. } \\
\text { Necesidad de depuración } \\
\text { extrarrenal no necesitada } \\
\text { previamente }\end{array}$ \\
\hline $\begin{array}{l}\text { Fracaso hematológico } \\
\text { (Presencia de uno o } \\
\text { más de lo siguiente) }\end{array}$ & $\begin{array}{l}\text { Presencia de CID, hematócrito } \\
<25 \%, \text { hemoglobina }<8 \mathrm{~g} / \%, \\
\text { plaquetopenia }<40.000 / \mathrm{ml}\end{array}$ \\
\hline Fracaso neurológico & $\begin{array}{l}\text { Escala de coma de Glasgow de } \\
\text { menos de ocho puntos } \sin ^{\wedge} \\
\text { sedantes }\end{array}$ \\
\hline
\end{tabular}

CID: coagulación intravascular diseminada.

tes al año, el $58 \%$ pertenecían al grupo A, el 30,6\% al B y sólo un $11 \%$ al C. El 46,7\% habían recuperado su CDVp, el 28,62\% la mejoraron y sólo el $24,65 \%$ la empeoraron. Sin embargo, sólo el $36 \%$ de los supervivientes menores de 65 años trabajaban al año, mientras que previamente lo hacían el $71 \%$ (de los cuales sólo la mitad se reincorporó al trabajo).

El coste de los pacientes según su evolución o categoría de CDV se muestran en la tabla 3 . El coste medio en UMI de los pacientes que fallecieron en la misma fue 2.285.660 (4.467.700) pts (13.769 [26.914] euros), en la sala de hospitalización previo a su ingreso en UMI fue de 247.249 (523.514) pts (1.489 [3.154] euros) y por consiguiente el coste medio total fue de 2.533.089 (4.534.078) pts (15.259 [27.314] euros). Asimismo, el coste medio en la UMI de los pacientes que fallecieron tras el alta de la misma fue de 2.199.375 (4.137.746 pts) (13.247 [24.926] euros), en la planta 1.057.875 $(1.225 .143,051)$ pts $(6.373$ [7.380] euros) y por tanto el coste medio total fue de 3.257 .250 (4.514.196) pts (19.622 [27.194] euros). El coste medio en UMI de un paciente en grupo A al año fue de 979.886,02 $(1.112 .448,24)$ pts $(5.903$ [6.701] euros), el coste hospitalario fue de 718.505,11 (695.512,77) pts (4.328 [4.190] euros) y el coste medio total de 1.698.391,14 $(1.404 .836,18)$ pesetas $(10.231$ [8.462] euros). Los pacientes del grupo B consumieron en UMI 1.788.495,45 $(2.178 .902,66)$ pesetas (10.774 [13.126] euros), en el hospital 991.207,79 $(1.196 .421,35)$ pts $(5.971$ [7.207] euros) y el coste medio total fue de $2.779 .703,24(2.701 .624,66)$ pts. (16.745 [16.275] euros). Finalmente, los pacientes del grupo $\mathrm{C}$ al año fueron los que consumieron mayores recursos, con un coste medio en UMI de
3.346.283,16 (4.236.960,26) pts (20.158 [25.524] euros), un coste hospitalario de 1.779 .375 $(2.161 .734,53)$ pts $(10.719$ [13.022] euros) y un coste medio total de $5.125 .658(5.759 .082,28)$ pts (30.877 [34.694] euros).

Estos gastos no incluyen el coste de los pacientes que fueron dados de alta a otro centro hospitalario y que fueron el $6 \%$ de los pacientes que estaban en grupo A al año, el 9,7\% de los B y el 35,7\% de los C.

En la tabla 4 se muestran los percentiles del gasto en UMI de los distintos grupos de pacientes y las diferencias entre ellos son estadísticamente significati$\operatorname{vas}(\mathrm{p}<0,01)$.

Como se puede apreciar en la figura 1, un escaso número de pacientes consumió un elevado coste. De los 843 pacientes estudiados, $196(23 \%)$ consumieron el $70 \%$ del total de recursos de la UMI y de estos pacientes los que fallecieron $(51 \%)$ y los que estaban en grupo $\mathrm{C}$ al año (15\%) consumieron el $72 \%$ de esos altos costes.

\section{DISCUSIÓN}

En la actualidad, los gastos que generan las UMI varían de unos países a otros. En EE.UU. se calculan entre el $0,8 \%$ y el $1 \%$ del producto nacional bruto $^{7,8}$, en Canadá es del $0,2 \%$, y en España es del $0,15 \%{ }^{10}$. En EE.UU. ${ }^{9,11}$ este coste supone el $15 \%$ $20 \%$ del presupuesto de los hospitales, en Canadá el $8 \%{ }^{9}$, en Noruega el 3\% ${ }^{12}$ y en España el 5,6\% (estimado a partir de los datos publicados por el Ministerio de Sanidad y Consumo sobre el gasto sanitario del año 1993 y Perales y Rucabado ${ }^{10}$ ). Parece evidente que el volumen de pacientes que ingresan en una UMI es cada vez más numeroso ${ }^{9}$, con un coste económico difícilmente sostenible y por ello es fundamental hacer una valoración de la eficiencia de estas unidades. En términos generales, la eficacia de nuestra unidad estaría probablemente demostrada ya que hemos conseguido una supervivencia al año del $60 \%$ y una mejoría o recuperación de la CDVa en el $75 \%$ de los supervivientes. Es muy posible que una gran proporción de estos pacientes hubieran fallecido si no hubieran ingresado en la UMI.

Para analizar la eficiencia debemos analizar el coste. Nuestra UMI consumió 1.520 millones de pesetas $(9,16$ millones de euros) durante el período de estudio y ello supuso el $11 \%$ de los gastos del hospital en ese período. Los altos costes asociados a las UMI, se deben tanto a la alta tecnología que conlleva como al tipo de patología ${ }^{13}$, y sobre todo a las altas estancias de los pacientes más graves ${ }^{14}$. Por ello nosotros hemos querido reflejar los costes, al igual que otros autores ${ }^{8,15,16}$ en términos de días de estancia.

Si comparamos el uso de recursos en pacientes supervivientes y no supervivientes es preciso tener en cuenta el período total de hospitalización y no sólo la estancia en UMI, puesto que los resultados de la evolución en la planta también forman parte de sus resultados. Como se muestra en la tabla 3, los pacientes que provocaron más gasto en la UMI fue- 
Apéndice 1: Encuesta de calidad de vida (EuroQOL Instrument ${ }^{5,6}$ modificado)



A continuación mostramos la clasificación en categorías de calidad de vida (CDV) a partir de la puntuación obtenida en la encuesta, la valoración subjetiva del paciente, la del médico y fundamentalmente la situación de dependencia de los demás para actividades cotidianas. Nótese que hay dimensiones de la CDV como el dolor que no determina el nivel de CDV, pudiendo haber tomado valores 1, 2, 3, para cada grupo.

\begin{tabular}{|c|c|c|c|c|c|}
\hline \multicolumn{6}{|c|}{ CATEGORÍA A. Gozan de buen estado de salud } \\
\hline$M=1$ & $M=1$ & $M=1$ & \multicolumn{2}{|c|}{$M=1$} & $M=1$ \\
\hline$A=1$ & $A=1$ & $A=1$ & \multicolumn{2}{|c|}{$A=1$} & $A=1$ \\
\hline $\mathrm{AC}=1$ & $\mathrm{AC}=1$ & $\mathrm{AC}=1$ & \multicolumn{2}{|c|}{$\mathrm{AC}=2$} & $\mathrm{AC}=1$ \\
\hline $\mathrm{AD}=1$ & $\mathrm{AD}=2$ & $\mathrm{AD}=1$ & \multicolumn{2}{|c|}{$\mathrm{AD}=1$} & $\mathrm{AD}=2$ \\
\hline $\mathrm{DI}=1$ & $\mathrm{DI}=2$ & $\mathrm{DI}=2$ & \multicolumn{2}{|c|}{$\mathrm{DI}=1$} & $\mathrm{DI}=1$ \\
\hline \multicolumn{6}{|c|}{$\begin{array}{l}\text { CATEGORÍA B. Tienen alguna discapacidad pero viven } \\
\text { independientes }\end{array}$} \\
\hline$M=2$ & $\mathrm{M}=1$ & $M=1$ & $M=1$ & $M=1$ & $M=1$ \\
\hline$A=1$ & $A=2$ & $A=1$ & $A=1$ & $A=1$ & $A=1$ \\
\hline $\mathrm{AC}=1$ & $\mathrm{AC}=1$ & $\mathrm{AC}=2$ & $\mathrm{AC}=1$ & $\mathrm{AC}=1$ & $\mathrm{AC}=3$ \\
\hline $\mathrm{AD}=1,2,3$ & $\mathrm{AD}=1,2,3$ & $\mathrm{AD}=2,3$ & $\mathrm{AD}=1$ & $\mathrm{AD}=3$ & $\mathrm{AD}=1$ \\
\hline $\mathrm{DI}=1,2,3$ & $\mathrm{DI}=1,2,3$ & $\mathrm{DI}=2,3$ & $\mathrm{DI}=3$ & $\mathrm{DI}=1$ & $\mathrm{DI}=1$ \\
\hline \multicolumn{6}{|c|}{$\begin{array}{l}\text { CATEGORÍA C. Discapacidades severas y dependen de } \\
\text { los demás para sobrevivir }\end{array}$} \\
\hline \multicolumn{3}{|c|}{$M=3$} & \multicolumn{3}{|c|}{$M=1,2$} \\
\hline \multicolumn{3}{|c|}{$A=3$} & \multicolumn{2}{|c|}{$\mathrm{A}=3$} & \\
\hline \multicolumn{3}{|c|}{$\mathrm{AC}=3$} & \multicolumn{2}{|c|}{$\mathrm{AC}=1,2,3$} & \\
\hline \multicolumn{3}{|c|}{$\mathrm{AD}=1,2,3$} & \multicolumn{2}{|c|}{$\mathrm{AD}=1,2,3$} & \\
\hline \multicolumn{3}{|c|}{$\mathrm{DI}=1,2,3$} & \multicolumn{2}{|c|}{$\mathrm{DI}=1,2,3$} & \\
\hline
\end{tabular}


TABLA 3. Distribución de los costes en la población estudiada

\begin{tabular}{|c|c|c|c|c|c|}
\hline & \multirow{2}{*}{$\begin{array}{l}\text { Fallecidos } \\
\text { en UMI }\end{array}$} & \multirow{2}{*}{$\begin{array}{l}\text { Fallecidos } \\
\text { Post-UMI }\end{array}$} & \multicolumn{3}{|c|}{ Categoría de calidad de vida al año } \\
\hline & & & A & B & $\mathrm{C}$ \\
\hline $\begin{array}{l}\text { N. }{ }^{\circ} \text { paciente } \\
\text { (\% del total) }\end{array}$ & $\begin{array}{c}212 \\
25 \%\end{array}$ & $\begin{array}{c}128 \\
20,2 \%\end{array}$ & $\begin{array}{c}293 \\
34,7 \%\end{array}$ & $\begin{array}{c}154 \\
18,26 \%\end{array}$ & $\begin{array}{r}56 \\
6,64 \%\end{array}$ \\
\hline $\begin{array}{l}\sum \text { Coste UMI (pts) } \\
\text { (\% del total) } \\
\text { Euros }\end{array}$ & $\begin{array}{l}484.560 .000 \\
30 \% \\
2.919 .040\end{array}$ & $\begin{array}{c}281.520 .000 \\
17,54 \% \\
1.695 .900\end{array}$ & $\begin{array}{c}287.640 .000 \\
18 \% \\
1.732 .770\end{array}$ & $\begin{array}{c}275.940 .000 \\
17,21 \% \\
1.662 .290\end{array}$ & $\begin{array}{r}187.740 .000 \\
11,7 \% \\
1.130 .960\end{array}$ \\
\hline $\begin{array}{l}\sum_{\text {Euros }} \text { Coste total (pts): } \\
\end{array}$ & $\begin{array}{c}537.015 .000 \\
3.235 .030\end{array}$ & $\begin{array}{c}417.928 .000 \\
2.511 .610\end{array}$ & $\begin{array}{l}498.162 .000 \\
3.000 .980\end{array}$ & $\begin{array}{c}428.586 .000 \\
2.581 .840\end{array}$ & $\begin{array}{r}287.385 .000 \\
1.731 .230\end{array}$ \\
\hline $\begin{array}{l}\text { Coste UMI: media (pts) } \\
\text { DE } \\
\text { Euros }\end{array}$ & $\begin{array}{c}2.285 .660 \\
4.467 .700 \\
13.769(26.913)\end{array}$ & $\begin{array}{c}2.199 .375 \\
4.137 .746 \\
13.249(24.926)\end{array}$ & $\begin{array}{c}970.886 \\
1.112 .448 \\
5.848(6.701)\end{array}$ & $\begin{array}{c}1.788 .497 \\
2.178 .902 \\
10.774(13.126)\end{array}$ & $\begin{array}{r}3.346 .283 \\
4.236 .960 \\
20.158(25.524)\end{array}$ \\
\hline $\begin{array}{l}\text { Coste planta: media (pts) } \\
\text { DE } \\
\text { Euros }\end{array}$ & $\begin{array}{c}247.249 \\
523.514 \\
1.489(3.153)\end{array}$ & $\begin{array}{c}1.057 .875 \\
1.225 .143 \\
6.373(7.380)\end{array}$ & $\begin{array}{c}718.505 \\
695.512 \\
4.328(4.190)\end{array}$ & $\begin{array}{c}991.207 \\
1.196 .421 \\
5.971(7.207)\end{array}$ & $\begin{array}{r}1.779 .375 \\
2.161 .734 \\
10.719(13.022)\end{array}$ \\
\hline $\begin{array}{l}\text { Coste total: media (pts) } \\
\text { DE } \\
\text { Euros }\end{array}$ & $\begin{array}{c}2.533 .089 \\
4.534 .078 \\
15.259(27.313)\end{array}$ & $\begin{array}{c}3.257 .250 \\
4.514 .196 \\
19.622(27.194)\end{array}$ & $\begin{array}{c}1.698 .391 \\
1.404 .836 \\
10.232(8.463)\end{array}$ & $\begin{array}{c}2.779 .703 \\
2.701 .624 \\
16.745(16.275)\end{array}$ & $\begin{array}{r}5.125 .658 \\
5.759 .082 \\
30.877(34.693)\end{array}$ \\
\hline
\end{tabular}

En la primera fila se muestran el número de pacientes de cada grupo y su porcentaje en relación al total de pacientes de Unidad de Medicina Intensiva (UMI). En la segunda fila se muestra la suma del coste de UMI en pesetas de todos los pacientes según su evolución o calidad de vida y porcentaje en relación al coste total de UMI. $\sum$ Coste total : $\Sigma$ Coste UMI + $\Sigma$ Coste planta hospitalización covencional; DE: desviación estándar.

ron los fallecidos en la misma, que consumieron el $30 \%$ de sus recursos. Si consideramos que los fallecidos post-UMI consumieron el $17,5 \%$ del presupuesto y los pacientes que quedaron con discapacidad severa el $11,7 \%$, podríamos concluir que el $60 \%$ del coste de la UMI se invirtió en tratamiento inútil. En líneas generales, los pacientes que más recursos consumieron fueron los pacientes de grupo $\mathrm{C}$, con un coste medio total aproximado de cinco millones de pesetas durante su estancia en el hospital al que habría que añadir los costes que generaron el $38 \%$ de estos pacientes que tuvieron que ser ingresados en centros para crónicos, los debidos a reingresos en ese año en el mismo hospital (el $28,5 \%$ de los pacientes con una estancia media de 23 [64 días], y los debidos a la dependencia física de otra persona para las actividades básicas de la vida cotidiana.

Los pacientes que menos recursos consumieron fueron los que al año presentaban una CDV A, y la diferencia con el resto de los grupos de pacientes fue estadísticamente significativa. La eficacia y efi- ciencia en el tratamiento de este grupo de pacientes estarían demostradas e incluso los altos costes invertidos en los pacientes del grupo B.

Como se refleja en la literatura ${ }^{8,17-20}$ un escaso número de pacientes consumen un gran porcentaje de recursos, y en nuestro caso el $23 \%$ de los pacientes consumió el $70 \%$ de los recursos de UMI. El $72 \%$ de estos altos costes lo consumieron los fallecidos y los que tenían una mala CDV al año. El coste de un paciente que fallece puede ser de 2 a 6,9 veces superior al coste de un superviviente ${ }^{8,17,21-26}$, y aumenta cuando el pronóstico es incierto. Bamberger et $\mathrm{al}^{24}$ calcularon el gasto en laboratorio y en radiografías que produjeron los pacientes 48 horas antes de morir en la UMI y comprobaron que en los pacientes a los que se les había pronosticado la muerte, y por tanto se limitó el tratamiento o se dio orden de no resucitar los gastos fueron significativamente menores. También Detsky et $\mathrm{al}^{21}$ observaron que sus pacientes fallecidos produjeron un mayor coste en su unidad y éste fue aún mayor cuando el pronóstico fue incierto. Oye y Bellamy ${ }^{8}$ también encontraron

TABLA 4. Percentiles del coste de la población estudiada

\begin{tabular}{|c|c|c|c|c|c|c|c|}
\hline Percentil & 5 & 10 & 25 & 50 & 75 & 90 & 95 \\
\hline \multirow[t]{2}{*}{ Fallecido UMI } & 180 & 180 & 360 & 900 & 2.700 & 5.580 & 7.380 \\
\hline & 1.084 & 1.084 & 2.168 & 5.420 & 16.270 & 33.610 & 44.460 \\
\hline \multirow[t]{2}{*}{ Fallecido post-UMI } & 360 & 360 & 540 & 900 & 2.475 & 4.410 & 6.615 \\
\hline & 2.168 & 2.168 & 3.252 & 5.420 & 14.910 & 26.570 & 39.850 \\
\hline \multirow[t]{2}{*}{ A } & 360 & 360 & 360 & 540 & 900 & 1.980 & 3.240 \\
\hline & 2.168 & 2.168 & 2.168 & 3.252 & 5.420 & 11.930 & 19.520 \\
\hline \multirow[t]{2}{*}{ B } & 360 & 360 & 540 & 900 & 2.160 & 4.320 & 7.470 \\
\hline & 2.168 & 2.168 & 3.252 & 5.420 & 13.010 & 26.020 & 45.000 \\
\hline \multirow[t]{2}{*}{$\mathrm{C}$} & 360 & 360 & 540 & 2.250 & 4.500 & 8.622 & 14.508 \\
\hline & 2.168 & 2.168 & 3.252 & 13.550 & 27.110 & 51.940 & 87.400 \\
\hline
\end{tabular}

El primer valor corresponde a pesetas y está multiplicado por $10^{3}$ y el segundo valor se expresa en euros. Obsérvese como en el percentil (P)50 la mayoría de pacientes consumieron 900.000 pesetas, salvo los pacientes del grupo A, y los de C. El 50\% de los pacientes fallecidos en UMI lo hace precozmente; UMI: Unidad de Medicina Intensiva. 


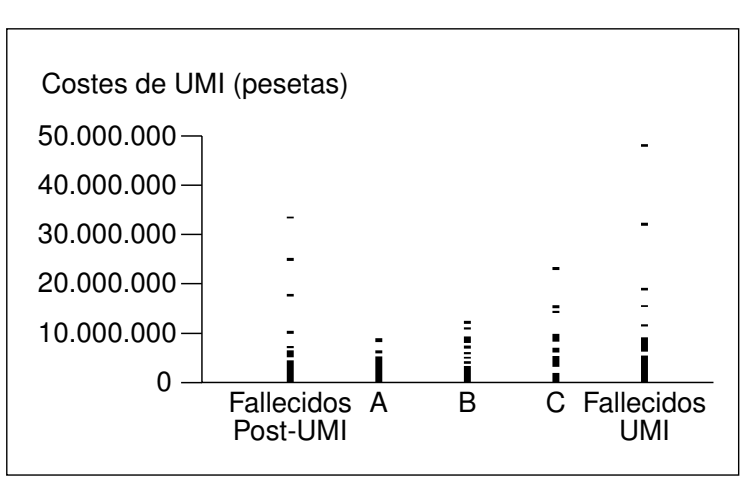

Fig. 1. Representación de los pacientes según su coste. En el eje de abscisas se muestran los pacientes agrupados por su evolución y en el de ordenadas el coste de Unidad de Medicina Intensiva (UMI) en pesetas. Cada punto representa un paciente. Nótese como la mayoría de los pacientes se agrupan por debajo de los 2,5 millones de pesetas y muy pocos sobrepasan los 7,5 millones de pesetas.

que sólo el $8 \%$ de sus pacientes provocaban gastos muy superiores al resto y que el $70 \%$ de estos gastos lo consumían los pacientes que fallecieron $(10 \%$ de los pacientes). Nuestros resultados no concuerdan exactamente con lo dicho anteriormente, ya que los pacientes que fallecieron (40\%) sólo consumieron el $47 \%$ del total de recursos de nuestra unidad. Esto es debido a que la mitad de nuestros pacientes fallecidos lo hicieron en los primeros cinco días de su estancia en la UMI, consumiendo como máximo 900.000 pesetas (5.422 euros) (tabla 4). Esto pudiera significar que en nuestra unidad se limitó el tratamiento precozmente en un mayor número de pacientes o que eran enfermos más graves y no pudimos mantenerlos con vida durante tanto tiempo como en los estudios que preceden. También es evidente que valorar los costes según la estancia es imperfecto, puesto que no valora el coste específico de cada paciente según su nivel de tratamiento. No obstante, si más de la mitad de nuestro gasto se produjo en los enfermos que fallecieron, este gasto inútil podría evitarse si fuéramos capaces de asegurar con absoluta certeza los pacientes que van a fallecer. Esto no ha sido posible hacerlo con ninguno de los scores de gravedad conocidos y por ello ninguno de ellos puede ser utilizado en la toma de decisiones individuales. Podríamos pensar que este gasto inútil se debería invertir en otros pacientes o en otras intervenciones de demostrada eficacia como podría ser una intervención de cataratas o una campaña de vacunación, pero el tema es más complejo y desde el punto de vista del clínico una distribución de los recursos basándose únicamente en criterios económicos no parece lícita. Sería la opinión pública la que tendría que intervenir en su distribución procurando el beneficio de la comunidad. El debate de la toma de decisiones es muy complicado y si fuera realizado entre los ciudadanos las respuestas serían diversas y probablemente desconcertantes ${ }^{27}$. Quizá deberían hacerse encuestas multitudinarias sobre estos problemas para concienciar a la población de los mis- mos y distribuir los recursos según las preferencias de los ciudadanos. No obstante, cada clínico debería administrar los recursos entre aquellos pacientes que más se beneficien, pero sin olvidar el principio de justicia y equidad ${ }^{28}$.

A pesar de ello, el tratamiento aplicado una vez ingresado el paciente en la UMI es difícil de racionalizar por los problemas ético-morales-legales que conlleva y sólo el reconocimiento del tormento emocional que el tratamiento prolongado y sin esperanza causa a familiares, médicos y enfermería y el coste sumamente elevado en detrimento de otros pacientes, parecen ejercer alguna influencia en la racionalización de los recursos limitados. La limitación del tratamiento en los enfermos sin expectativas de alcanzar la recuperación con un nivel de vida aceptable constituye un hecho reconocido en todo el mundo ${ }^{29,30}$ pero cuando el enfermo ya está ingresado, éste conserva la prioridad para recibir todas las medidas aunque sus posibilidades de recuperación sean escasas o inferiores a las de otros pacientes que aún no han ingresado ${ }^{28}$. Esto podría ser interpretado en el sentido de que el principio de justicia tiene escaso valor en el caso de tener que decidir qué pacientes deben ser tratados en las situaciones de escasos recursos y que sólo las razones de la autonomía del paciente, si se considera capacitado para ello, pueden hacer cambiar este tipo de decisiones ${ }^{28}$. Se deberían establecer guías éticas de actuación basadas en estudios científicos y la experiencia de los médicos, puesto que la selección subjetiva debe sustituirse por sistemas objetivos formales que nos ayuden a distribuir adecuadamente los recursos de $\mathrm{UMI}^{30-32}$.

Evidentemente a la hora de tomar decisiones económicas explícitas, los juicios aportados a la Administración tienen que ser más precisos y exigentes que los sistemas evaluativos que aportan información general del estado de salud en forma de perfil. Para comparar los costes con el beneficio obtenido en términos de CDV se necesita utilizar valores numéricos $\left(\mathrm{QALY}^{6}\right)$. Este tema no está suficientemente desarrollado en Medicina Intensiva.

El concepto de CDV es fundamental a la hora de establecer prioridades puesto que los recursos son limitados y nuestro esfuerzo debe ir encaminado a lograr una vida digna. Lógicamente se deberían concentrar los recursos en los tratamientos que produzcan mayor bien y dado que los pacientes que más se benefician son los pacientes de grupo A, que son además los que menos recursos consumen, cuanto mayor sea el número de pacientes de este grupo ingresados más eficiente será nuestra labor. ¿Deberíamos ingresar, por tanto, solamente a estos pacientes? No sería razonable que el intensivista tuviera que tomar esta decisión, pero tampoco el economista o el político podrá tomarla, puesto que le faltan conocimientos médicos para ello. Las decisiones que tomen los políticos deben estar perfectamente fundamentadas en las valoraciones de los clínicos. Como puede verse el problema es de difícil solución, y se hace mucho más inquietante si nos fija- 
mos en la fatídica "lista de espera" "33, es decir, en los pacientes que no pueden acceder a una cirugía de demostrada eficacia y eficiencia, a causa de que otro paciente con otra patología, cuyo tratamiento no es tan eficaz ni tan eficiente, está ocupando su lugar en la UMI.

Existe, por tanto, un grupo de pacientes cuyo tratamiento intensivo sólo alarga el proceso de muerte (pero sólo posteriormente a su ingreso podremos confirmar nuestras sospechas sobre este hecho) y otro grupo que ingresan en las unidades sólo para monitorización, con escaso riesgo de morir o sufrir complicaciones $^{8}$ y en nuestra opinión su hospitalización en áreas apropiadas para esta función y distintas del área propia de Medicina Intensiva mejoraría la relación coste-efectividad de la misma.

También debería tenerse en cuenta que el tratamiento aplicado en las UMI proporciona a todos los enfermos un riesgo de complicaciones añadidas y por tanto habría que valorar en cada paciente el riesgo-beneficio de cada tratamiento aplicado.

El análisis de la mortalidad hospitalaria y de la CDV aparece como un valioso instrumento para la planificación y gestión de los recursos de los hospitales $^{6,34}$. El problema es que para poder planificar el gasto sanitario de una forma más eficiente tendríamos que predecir con una certeza absoluta el desenlace de cada paciente y actuar precozmente en consecuencia, pero esto hoy en día no es posible ya que no existen escalas predictivas para este fin.

El problema es tan difícil que realmente la toma de estas decisiones varía incluso de unos hospitales a otros y muchas veces está sujeto a la subjetividad del médico ${ }^{35}$. En estudios recientes ${ }^{30,35,36}$ la limitación de tratamiento o ingreso en las UMI se ha realizado en el 64\%-90\% de los fallecidos y ello implica una sensibilización progresiva en los problemas de efectividad y mejora de los cuidados. Para poder disminuir los costes debemos tomar decisiones correctas y por tanto para mejorar la eficiencia, sin disminuir la eficacia, se necesita fijar un pronóstico certero desde el momento del ingreso ya que se sabe que los gastos aumentan cuanto más incierto es el pronóstico ${ }^{8,21,24}$.

Basándonos en el principio de equidad (todos los pacientes tienen iguales derechos a iguales tratamientos) lo más ético es abogar por el principio de autonomía de cada paciente o su familia e informarles adecuadamente para llegar a un acuerdo en las decisiones a tomar ${ }^{37,38}$.

En conclusión podríamos decir que nuestros resultados fueron aceptables ya que el $89 \%$ de los supervivientes al año eran capaces de vivir de modo independiente y sólo un $25 \%$ había empeorado su CDV previa. Sin embargo las ganancias sociales obtenidas fueron más dudosas, ya que sólo el $36 \%$ de los pacientes menores de 65 años trabajaban al año del alta de UMI. Los determinantes últimos de esta situación no los conocemos, pero probablemente habrá que buscarlos en factores socioculturales y en el tipo de trabajo y motivación por el mismo de cada paciente.
En cuanto a la utilización de recursos se refiere, el $70 \%$ del coste lo consumieron el $23 \%$ de los pacientes y éstos fueron los que fallecieron o quedaron con peor CDV. Esto no podrá evitarse mientras no mejoren nuestras predicciones de supervivencia y CDV y por tanto no es razonable pedir que el médico seleccione aquellos pacientes que deben ser tratados o no, basándose sólo en criterios económicos. Tampoco los políticos deben dictar normas en este sentido y según nuestra opinión, debería ser la sociedad la que tomara la decisión de cómo distribuir los recursos y de si a ciertos pacientes debe limitarse o no la posibilidad de tratamiento. Mientras esto no se establezca, al intensivista se le plantean serios problemas cuando tiene que realizar una selección de los pacientes por falta de recursos en la unidad y lo único que se nos puede pedir es ética, justicia y equidad, sabiendo que lo fundamental para poder disminuir los costes y aumentar la eficiencia de nuestras UMI, sería estratificar correctamente el riesgo de muerte mejorando los índices pronósticos.

\section{BIBLIOGRAFÍA}

1. Ruíz J. Identificación de las relaciones de agencia en Medicina Intensiva y su implicación ética y económica. En: Net A ed. Ética y Costes en Medicina Intensiva. Barcelona: Springer, 1996; 13: 116-39.

2. Knaus W, Draper E, Warner DP, Zimmerman J. Prognosis In Acute Organ-System Failure. Ann Surg 1985; 202: 685-693.

3. Marshall J, Cook DJ, Christou NV, Bernard GR, Sprung CL, Sibbald WJ. Multiple Organ Dysfunction Score: A Reliable Descriptor of a Complex Clinical Outcome. Crit Care Med 1995; 23: $1.638-1.652$.

4. Jorda Marcos R, Abizanda Campos R, Verduras Ruiz MA, Revuelta Rabasa P, Chama Barrientos A, Abadal Centellas JM. Mortalidad en el Fracaso Multiorgánico (FMO). Med Intensiva 1985; 9: 220-222.

5. EuroQoL Group. Euroqol a New Facility for the Measurement of Health- Related Quality of Life. Health Policy 1990; 16: 199-208.

6. Williams A. The Role of the Euroqol Instrument in QALY Calculations. Discussion Paper 130. The University of York, 1995.

7. Reis Miranda D. Una aproximación al control de calidad de las unidades de cuidados intensivos. En: Europa. Med Intensiva 1991; 15: 319-321.

8. Oye RK, Bellamy PE. Patterns of Resource Consumption in Medical Intensive Care. Chest 1991; 99: 685-689.

9. Jacobs P, Noseworthy TW. National Estimates of Intensive Care Utilization and Cost: Canada and United States. Crit Care Med 1990; 18: 1.282-1.286.

10. Perales N, Rucabado L. La Medicina intensiva en la crisis de los sistemas sanitarios. El reto de la eficiencia: planificación y regionalización. En: La UMI como Centro de responsabilidad. Planificacion y Control. Madrid: IDEPSA 1991; 37-65.

11. Donabedian A. Una aproximacion a la monitirización de la calidad asistencial (Primera Parte) Control de calidad asistencial, 1991; 6. 1: 1-6.

12. Les $R$, Smith-Erucgseb $N$, Lind $B$. Intensive Care: Cost And Benefit. Acta Anaesthesiol Scand 1987; 31 (Supl). 84: 3-19.

13. Sage WM, Hurst $C$, Silverman J, Bortz W. Intensive Care for The Elderly: Outcome of Elective and Nonelective Admissions. J Am Geriatr Soc 1987; 35: 312-318.

14. Chelluri L, Grenvik A, Silverman M. Intensive Care for Critically Ill Elderly: Mortality, Cost and Quality of Life. Review of The Literature. Arch Intern Med 1995; 155: 1.013-1.022.

15. Becker G, Strauch G, Saranchak H. Outcome and Cost of Prolonged in the Surgical Intensive Care Unit. Arch Surg 1984; 119: $1.338-1.342$. 
16. Slatyer MA, James OF, Moore PG, Leeder SRl. Cost, Severity of Illness and Outcome in Intensive Care. Anaesth Intens Care 1986; 14: 381-389.

17. Bohigas Ll. El Coste de la Muerte. En: Net A, ed. Ética y Costes en Medicina Intensiva. Barcelona: Springer 1996; 37-44.

18. IBERN. La distribución de los costes sanitarios per cápita y el control del riesgo. XV Jornadas de Economía de la Salud, Santiago (Junio) 1994.

19. Berk ML, Monheit AC. The Concentration of Health Expeditures: An Update. Affairs (Winter) 1992: 144-149.

20. Mizrahi An, Mizrahi AR. Debours et Depenses Medicales Selon L'âge Et Le Sexe. Credes (Paris) 1985.

21. Detsky AS, Stricker S, Mulley A, Thibault GE. Prognosis, Survival and the Expenditure of Hospital Resources for Patients in an Intensive-Care Unit. N Engl J Med 1981; 305: 667672.

22. Byrick RJ, Mindorff C, McKee L, Mdge B. Cost-Effectiveness of Intensive Care For Respiratory Failure Patiens. Crit Care Med 1980; 8: 332-337.

23. Chassin MR. Costs and Outcomes of Medical Intensive Care. Med Care 1982; 20:165-179.

24. Bamberger K, Maniscalco-Theberge M, Pearl R, Jaques D. Death and Dollars: the Cost of Dying in the Surgical Intensive Care Unit. J Trauma 1996; 40: 39-41.

25. Ridley S, Biggam M. A Cost-Utility Analysis of Intensive Therapy. II: Quality of Life in Survivors. Anaesthesia 1994; 49: 192-196.

26. Ridley S, Biggam M, Stone $P$. The Cost of Intensive Care; A Descripcion of Methodology and Initial Results. Anaesthesia 1991; 46: 523-530.

27. Roberts C. Implications for Clinical Practice. En: Measuring Health: A Practical Aproach. London: John Wiley, 1988; 245-260.
28. Gómez Rubi JA. La Influencia de la escasez de recursos en la limitacion del esfuerzo terapéutico. En: Net, ed. Ética y costes en medicina intensiva. Barcelona: Springer 1996; 97 103.

29. Manzano JL. Limitación del tratamiento a pacientes críticos. Med Clin (Barc) 1994; 102: 540-542.

30. Wood G, Martin E. Withholding and Withdrawing LifeSustaining Therapy in a Canadian Intensive Care Unit. Can $\mathbf{J}$ Anaesth 1995; 42: 186-191.

31. Kalb PE, Miller DH. Utilization Strategies for Intensive Care Units. JAMA 1989; 261: 2.389-2.395.

32. Solsona JF, Abizanda R. Hacia la elaboración de criterios de ingreso en los servicios de medicina intensiva. Med Intensiva 1998; 22: 72-73.

33. Williams A. Applications in Management. En: Measuring Health: a Practical Approach. Teeling Smith, eds. London: John Wiley \& Sons, 1988; 225-244.

34. Williams A. El problema del establecimiento de prioridades en sanidad. En: Net A, eds. Ética y costes en medicina intensiva. Barcelona: Springer 1996; 3: 21-36.

35. Keenan SP, Busch KD, Chen LM, Esmail R, Inman KJ, Sibbald WJ. Wihtdrawal and Withholding of Life Support in the Intensive Care Unit: a comparison of Teaching and Community Hospitals. Crit Care Med 1998; 26: 245-251.

36. Prendergast TJ, Luce JM. Increasing Incidence of Withholding and Withdrawal of Life Support from the critically Ill. Am J Respir Crit Care Med 1997; 155: 15-20.

37. Dowdy M, Robertson C, Bander J. A Study of Proactive Ethics Consultation for Critically and Terminally Ill Patients with Extended Lengths of Stay. Crit Care Med 1998; 26: 252-259.

38. Vincent JL. European Attitudes Towards Ethical Problems in Intensive Care Medicine Results of an Ethical Questionnaire. Intensive Care Med 1990; 16: 256-264 